\section{Cahiers de Narratologie}

Analyse et théorie narratives

$35 \mid 2019$

Le style comme événement

\title{
La figuralité comme événement de style : l'exemple de la métonymie
}

\section{Marc Bonhomme}

\section{(2) OpenEdition \\ 12 Journals}

Electronic version

URL: https://journals.openedition.org/narratologie/9286

DOI: 10.4000/narratologie.9286

ISSN: 1765-307X

Publisher

LIRCES

\section{Electronic reference}

Marc Bonhomme, "La figuralité comme événement de style : l'exemple de la métonymie", Cahiers de Narratologie [Online], 35 | 2019, Online since 03 septembre 2019, connection on 28 juin 2022. URL: http://journals.openedition.org/narratologie/9286 ; DOI: https://doi.org/10.4000/narratologie.9286

This text was automatically generated on 30 September 2020 .

Article L.111-1 du Code de la propriété intellectuelle. 


\title{
La figuralité comme événement de style : l'exemple de la métonymie
}

\author{
Marc Bonhomme
}

1 Composantes essentielles du style littéraire, les figures du discours ont majoritairement été étudiées suivant une approche objectivante qui les détache de leur contexte et de la vie effective des œuvres. Elles sont alors vues comme des structures autonomes préexistant à leur mise en texte et comme des réalités "sèches " au fonctionnement stabilisé dans le temps. Cette approche caractérise les théories formalistes sur les figures, de Fouquelin (1555) au Groupe $\mu$ (1970), qui se bornent généralement à une description de leurs principaux traits, exemples à l'appui. Elle est également avérée dans les typologies rhétoriques de la figuralité, comme celles de Quintilien (rééd. 1978) ou de Fontanier (1821), avec leurs catégorisations figées. Cependant, parallèlement à cette conception que l'on peut qualifier d'ontologique, la figuralité a nourri une approche phénoménologique, centrée sur ses aspects discursifs et ses manifestations textuelles. Cette approche en acte des figures est notamment celle de Longin au III ${ }^{\mathrm{e}}$ siècle (rééd. 1993), qui les rattache à une rhétorique de l'enthousiasme, ou celle de Lamy (1675) pour lequel elles expriment les passions. Mais elle est surtout développée dans les recherches récentes, avec la mise en exergue des dimensions constructivistes (Tamba-Mecz 1981, Gardes Tamine 2011), argumentatives (Perelman et Olbrechts-Tyteca 1988, Plantin 2009), énonciatives (Détrie 2001, Rabatel 2008) et pragmatiques (Kerbrat-Orecchioni 1994, Bonhomme 2014) des figures.

2 La présente contribution s'inscrit dans une telle perspective phénoménologique, en reconsidérant la figuralité comme un événement de style indissociable de la production des textes littéraires. L'intérêt de cette conception est de dynamiser la vieille terminologie de «fait de style » et de penser les figures comme des processus participant, par leur créativité, à la personnalisation des œuvres d'art verbales. Mais devant les risques de fragmentation attachés aux études phénoménologiques de la figuralité, il importe d'appréhender celle-ci en s'appuyant sur un cadre modulaire, attentif à une approche intégrative de son hétérogénéité. Selon ce cadre modulaire, les figures comportent des points d'ancrage prédominants qui déterminent leur identité, tout en 
laissant le champ libre à des variations discursives qui concourent à leur spécificité dans chaque énoncé. De plus, un traitement modulaire des figures permet de prendre en compte, sans réductionnisme, leur multidimensionnalité et la complémentarité de leurs niveaux d'organisation dont la conjonction crée des effets de style pertinents ${ }^{1}$.

Afin d'étayer notre étude par un corpus délimité et homogène, nous nous concentrerons sur la figure représentative qu'est la métonymie, définie par ses décalages de dénomination entre des termes contigus dans un domaine notionnel ${ }^{2}$. Outre qu'elle forme l'un des principaux procédés attestés dans les textes littéraires, en particulier le récit réaliste comme l'a établi Jakobson ${ }^{3}$, elle offre l'avantage d'avoir été beaucoup moins analysée que sa figure jumelle, la métaphore. Par ailleurs, la modération supposée de son fonctionnement et la relative discrétion de ses effets stylistiques montrent paradoxalement qu'une figure même réputée sobre possède les propriétés déstabilisantes et significatives d'un événement.

4 Notre étude procèdera en deux temps. D'une part, nous verrons en quoi, lors de sa production, la métonymie constitue un événement de style disruptif dans les énoncés littéraires. Seront examinés les marques linguistiques locales de son action perturbatrice, ainsi que les effets plus globaux de cette dernière sur les séquences textuelles. D'autre part, nous évaluerons les répercussions des événements de style métonymiques produits sur leur réception, en explorant les procédures d'identification et d'interprétation mises en place à leur propos par les lecteurs. Au bout du compte, ces événements de style métonymiques se dévoileront dans toute leur chronogénèse, à travers les questionnements qu'ils suscitent et les réactions diversifiées auxquelles ils donnent lieu.

\section{La production métonymique : un événement de style disruptif}

5 Sous l'angle de sa production qui constitue un premier module d'analyse, la métonymie est un événement de style par le fait qu'elle apparaît comme une intrusion inopinée dans le déploiement des énoncés littéraires. Liée à l'actualisation de la figure par l'acte d'énonciation de son producteur ${ }^{4}$ et s'intégrant dans une phénoménologie de l'émergence 5 , cette intrusion contribue à la singularisation stylistique de ces énoncés, en introduisant une disruption en leur sein. Celle-ci présente trois grands traits qui concernent leurs sous-modules communicationnels, textuels et discursifs.

\section{Ruptures communicationnelles}

6 En premier lieu, la métonymie se manifeste par des ruptures communicationnelles à la surface des textes littéraires, comme l'illustrent ces deux exemples reposant sur une métonymie du signe ${ }^{6}$ :

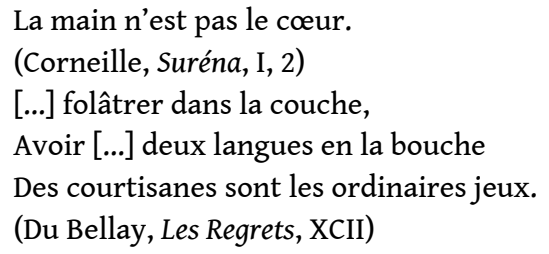

7 Envisagées d'un point de vue négatif, ces deux occurrences figurales violent ostensiblement plusieurs lois du discours. La première d'entre elles est un truisme, frappé 
du sceau de l'évidence, ce qui contrevient à la loi d'informativité formulée par Ducrot (1979), selon laquelle tout énoncé doit apporter un minimum de savoir nouveau à ses destinataires. La seconde occurrence "avoir [...] deux langues en la bouche» va à l'encontre d'au moins deux maximes théorisées par Grice (1979) : celles de qualité, en ce qu'elle exprime une contrevérité sur le corps humain, et celle de modalité, en ce qu'elle rend l'énoncé obscur.

En perturbant la cohérence communicationnelle des occurrences en jeu, ces violations produisent de l'imprévu qui les convertit en événements discursifs. Non seulement elles attirent l'attention des lecteurs sur elles, mais elles provoquent aussi leur surprise en désorientant leur horizon d'attente. Sur un autre plan, ces énoncés métonymiques répondent aux conditions de base du style selon Riffaterre (1971: 57), celles de l'« insertion d'un élément inattendu dans un pattern » et d'une "rupture qui modifie le contexte ». De surcroît, ils confirment l'idée émise par Meyer (2008: 256) que les figures sont des zones où « le texte se problématise », soulevant l'interrogation des lecteurs.

\section{Saillances textuelles}

Plus positivement, une figure comme la métonymie partage la densité et la dimension notable de l'événement ${ }^{7}$ par ses formations saillantes ou ses «conformations particulières » (Dumarsais 1967 : 767) qui se détachent sur le substrat du texte littéraire. Témoignant d'un épaississement de la substance langagière, la métonymie se concrétise dès lors par des structures stylistiques en relief qui individualisent chaque occurrence et qui fournissent un sens composite en une seule saisie. En résultent des configurations surdéterminées produites suivant diverses modalités.

D'un côté, la métonymie est à la source de densifications diégétiques qui resserrent la trame narrative du texte. Hétérogènes les uns par rapport aux autres en dehors du principe dynamique qui les relie, les pôles composant la diégèse finissent par se confondre à la suite de transferts métonymiques. Ainsi en est-il pour le produit (vêtement) et le but (deuil) :

La Félicie a sorti son deuil. Le deuil toujours prêt dans l'armoire.

(Giono, Le Grand Troupeau, p. 81)

ou pour l'instrument (klaxon) et l'agent (automobilistes) :

Derrière des claquesons râlaient.

(Queneau, Zazie dans le métro, p. 113).

12 D'un autre côté, la métonymie opère des amalgames notionnels dans les descriptions. Tantôt elle transfère l'une des propriétés terrestres d'un paysage sur un élément aérien qui lui est contigu :

Au milieu des arbres apparaît le poème des toits. [...] L'air est vert.

(Claudel, « Jardins », Connaissance de l'Est)

13 Tantôt elle unifie par le biais de l'hypallage ${ }^{8}$, dans un mouvement synesthésique entremêlant l'ouie et la vue, deux éléments terrestres coprésents :

Les amants séparés font des gestes hagards

Dans le silence blanc de l'hiver ${ }^{9}$

(Aragon, «Les amants séparés ", Le Crève-cœur)

14 Les figures apparentées à la métonymie que sont la synecdoque et la métalepse ${ }^{10}$ engendrent des configurations syncrétiques analogues. Par ses focalisations 
référentielles, la première concentre couramment une entité vivante, homme ou animal, sur l'une de ses parties distinctives :

Des centaines de millions de "pinces" sont en marche. Les crabes sortent de l'humidité de la forêt pour se jeter en direction des plages. (Moutot, Séquoias, p. 47)

Quant à la métalepse, ses transferts chronologiques lui permettent de fusionner le statut passé (prêtre) et le statut présent (pape) d'un individu en une seule assertion :

Le grand empereur Henri IV attendit trois jours [...] que le prêtre Grégoire VII daignât lui permettre de se mettre à genoux devant lui. (Voltaire, Questions sur les miracles, p. 287)

Apparaissant comme des événements stylistiques remarquables en raison de l'originalité de leurs configurations, ces occurrences métonymiques saillantes ont en outre une extension très flexible dans les énoncés. Le plus souvent, elles se distinguent par leur émergence singulière lorsqu'elles surgissent ponctuellement sur le dépli du texte. Ce faisant, elles forment un pic structural qui survient plus ou moins fortuitement dans le continuum du discours, à l'instar de cet exemple de Voltaire :

Nous allons chercher à la Chine de la terre comme si nous n'en avions point.

(" Chine (de la)», Dictionnaire philosophique)

Ici, la seule actualisation du nom "terre » suffit pour créer une incongruence dans l'énoncé, laquelle provient d'une métonymie de la matière pour désigner la porcelaine. Mais il arrive que le caractère frappant de la figure soit estompé par son entour textuel, notamment quand une métonymie du vêtement pour l'individu qui le porte émerge par ellipse après la non-reprise d'un terme antérieur :

Un bruit soudain me fit tourner la tête, il était occasionné par une troupe d'hommes vêtus de longues robes noires. [...] Voyez-vous, me dit ma conductrice, j'en use moins sévèrement avec les officiers qu'avec ces robes noires. Leur encens pourrait défigurer les bustes. (Diderot, Les Bijoux indiscrets, p. 154)

Les productions métonymiques présentent une extension plus ample lorsqu'elles se dévoilent à travers le surgissement conjoint de plusieurs polarités convergentes. Fondé sur une phénoménologie de la sériation, ce type de configuration tire sa figuralité de sa nature séquentielle et de l'ancrage prononcé du processus métonymique dans le tissu textuel. Il est particulièrement remarquable quand la sériation se fait évolutive. Dans ce cas, le texte s'agence autour d'un référent de base, mais celui-ci se voit traité selon plusieurs transferts métonymiques enchaînés. Le passage construit une saillance complexe, à la fois continue et discontinue, en filant une succession de désignations obliques sur le référent en question:

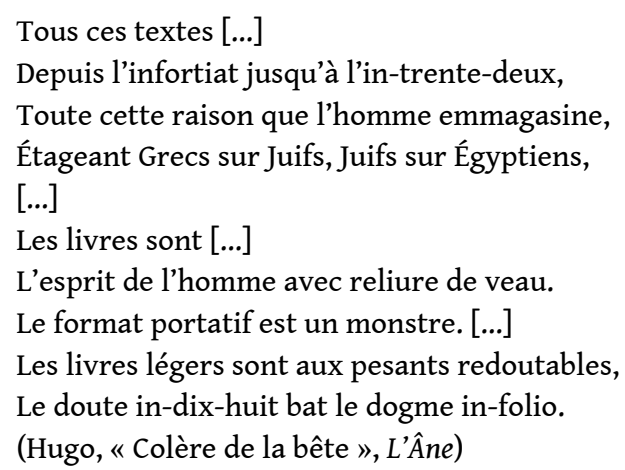

19 Dans cet extrait consacré au savoir accumulé par l'homme depuis l'Antiquité, le référent «livre(s)» est reprofilé au moyen de quatre transferts métonymiques: ceux de la dimension pour l'objet (in-trente-deux/livres, format/livres), du contenu pour le 
contenant (doute/livre, dogme/livre), de l'auteur pour l'œuvre (Grecs/livres, Juifs/livres, Égyptiens/livres) et de la cause pour l'effet (raison/livres, esprit/livres).

\section{Tensions discursives}

Plus globalement, l'émergence d'un événement de style métonymique dans un énoncé établit une tension discursive entre le pôle figural au premier plan et son cadre. En activant le pôle figural, le cadre forme en effet une partie intégrante du processus métonymique. De plus, comme l'a montré Riffaterre (1971), une telle confrontation entre un élément microcontextuel perturbateur et son environnement macrocontextuel fonctionne comme un stimulus stylistique appelant une lecture esthétique de l'énoncé. Les tensions métonymiques induisant cette lecture esthétique sont de trois ordres, ceuxci pouvant se combiner et être envisagés en terme de dominantes dans la communication textuelle.

21 Ces tensions sont cotextuelles lorsqu'elles surviennent dans l'environnement linguistique immédiat du pôle métonymique. Elles peuvent se fixer sur la graphie qui constitue la marque la plus immédiatement perceptible d'un événement métonymique. De la sorte, ce dernier est signalé par des guillemets :

Une jeune femme, l'avant-bras chargé d'une "semaine en or ", s'adressait à sa voisine.

(Ousmane, Le Docker noir, p. 82)

et par des italiques :

Je vis M. le premier président, madame de Soubise [...] et parmi tout cela cette sorte d'étourdis que vous appelez des talons rouges, et que j'eus bientôt congédiés. (Diderot, La Religieuse, p. 282)

Ces marquages graphiques introduisent une tension discursive entre le statut d'usage des énoncés encadrants et le statut de modalisation autonymique des formulations métonymiques insérées en leur $\operatorname{sein}^{11}$, lesquelles sont dues respectivement à des transferts par contiguïté Temps indiqué (semaine)/Produit (bracelet) et Produit (talon)/ Individus. Cette première tension se double d'une tension énonciative complémentaire : autant les énoncés encadrants sont dits par leurs énonciateurs, autant les formulations métonymiques isolées graphiquement sont citées par eux, avec une nuance entre les deux occurrences puisque les guillemets annoncent une distanciation et les italiques un soulignement. Plus fréquemment, les tensions métonymiques cotextuelles affectent l'agencement syntaxique des énoncés. Ainsi, au niveau de la détermination, l'injection d'un article partitif (+ discontinu) devant un nom abstrait (+ continu) provoque ipso facto une incongruité syntaxique dans sa construction :

Il se cogna dans de la jeunesse qui se préparait à consommer en ce café célèbre.

(Queneau, Les Derniers jours, p. 36)

Convertissant la dénotation unitaire du nom abstrait « jeunesse » en une partie d'un tout, l'incongruité engendrée met en lumière le fonctionnement métonymique de ce nom (Entité abstraite/Individus intégrés). De même, au niveau de l'organisation coréférentielle des énoncés, l'apparition d'un transfert métonymique crée souvent des disjonctions dans le marquage anaphorique du genre, à l'exemple de cet extrait du Neveu de Rameau (p. 105) de Diderot : 
Les vieilles perruques qui viennent là depuis trente à quarante ans tous les vendredis, au lieu de s'amuser comme ils ont fait par le passé, s'ennuient et baillent sans trop savoir pourquoi. Ils se le demandent et ne sauraient se répondre. textuel ne comporte aucune indication qui infirme cette impression. Mais le contexte référentiel de cet énoncé de Voltaire révèle un transfert métonymique par contiguïté géographique dans le nom propre «Phénicie ». En parlant de la Phénicie, Voltaire réfère en fait à la Palestine, faisant allusion à la déportation des Juifs à Babylone par Nabuchodonosor. Surgissant ainsi dans l'interface du texte et de la réalité historique, la métonymie Phénicie/Palestine produit une tension informative par son brouillage de la référence : la Phénicie n'est pas explicitement la Palestine, tout en l'étant implicitement. Cette tension informative s'accompagne d'une tension énonciative causée par le positionnement polémique de Voltaire dont le propos est de nier l'existence de la nation juive.

29 Les tensions attachées à la genèse d'une métonymie sont encore conceptuelles quand elles adviennent au contact d'une occurrence et de la mémoire discursive de ses producteurs ou de ses destinataires, à l'image de cette phrase tirée de la Correspondance de Flaubert :

J'ai reçu un autre cadeau : un livre du Faune et ce livre est charmant. (Lettre à sa nièce Caroline du 17 juin 1876)

Si cet énoncé ne contient pas de rupture interne sur les plans syntaxique ou sémantique, le syntagme « du Faune » est quelque peu obscur et il faut faire appel au savoir culturel partagé pour voir que cette formulation réfère métonymiquement à Stéphane Mallarmé par le biais de son poème "L'après-midi d'un Faune ». Apparaît dès lors une tension cognitive axée sur le terme « Faune » qui désigne discursivement un écrivain, sans perdre pour autant son acception première d'œuvre. De telles tensions cognitives sont très vivaces avec les métonymies à portée symbolique, comme dans cet exemple : 
Père [...] préférait aux socialistes le casque à pointe de là-bas.

(Queneau, «À quelques pas des gazomètres ", Chêne et chien)

les ruptures communicationnelles, les saillances textuelles et les tensions discursives qui lui sont propres et qui la singularisent dans les textes littéraires. Se pose à présent la question du statut stylistique de cette singularisation. Vaut-il mieux l'appréhender en recourant à une stylistique de l'écart ou de la variation? Une stylistique de l'écart, comme la pratique Cohen (1970) et qui correspond au traitement traditionnel de la métonymie (voir Fontanier 1821), offre l'avantage de faire des occurrences métonymiques que l'on a relevées des événements de style véritablement nouveaux puisqu'ils s'opposent, d'après cette approche, aux formes normées de la communication standard, dans une altérité intrinsèque par rapport à elle. Mais cette approche présente au moins deux difficultés. D’abord, elle présuppose une conception négative de la figuralité, la notion d'écart étant voisine de celle d'« anomalie » (Todorov 1967 : 108) par laquelle on a aussi défini les figures. Ensuite, faire des figures comme la métonymie des écarts revient à les couper des processus ordinaires du langage, ce qui n'est pas conforme à leur fonctionnement effectif. C'est pourquoi il est préférable d'étudier les occurrences métonymiques en s'appuyant sur une stylistique variationnelle, telle qu'elle est théorisée par Adam (1997) ou Herschberg-Pierrot (2005). Dans cette perspective, elles sont définissables comme des variations libres qui exploitent la plasticité de la langue et ses ramifications plus marginales pour produire un discours idiolectal adapté à chaque contexte. Sous cet angle, en tirant parti des transformations permises par la langue, les événements de style métonymiques ne sont pas absolument nouveaux par rapport à elle, mais ils le deviennent dans le cadre irréductible de chaque occurrence littéraire qui les prend en charge et qui les met en valeur selon ses besoins.

\section{La réception métonymique : un événement herméneutique de stylisation}

33 Événements de discours opacifiant les énoncés littéraires, les occurrences métonymiques n'ont encore qu'un statut stylistique limité tant qu'elles ne sont pas évaluées à travers leur phase de réception qui constitue un second module d'analyse, complémentaire à leur production. En effet, adressées aux lecteurs, leurs configurations disruptives sollicitent en creux l'activité métadiscursive de ces derniers pour être pleinement stylisées, c'est-àdire pour voir leurs apports fonctionnels et esthétiques mis en évidence ${ }^{13}$. Cette activité de stylisation apparaît comme un événement herméneutique par sa nature conjoncturelle, par ses évolutions au gré des lectures et par son incertitude, en ce qu'il est délicat d'en prévoir l'issue.

En face d'une occurrence métonymique, les lecteurs peuvent s'en tenir à un régime de réception empathique. Ce faisant, tout en ayant conscience de son caractère plus ou moins figural, ils en restent à une attitude infra-réflexive, se bornant à adhérer à 
l'immédiateté de ses effets. Par exemple, devant l'occurrence de Zola relevée précédemment : « la mort qui volait de toutes parts ", ils seront peut-être sensibles à ses effets dysphoriques et à la vision dramatisante qu'elle établit sur la guerre. Mais fondée sur une représentation impressive, cette réception empathique suscite une stylisation encore approximative des occurrences métonymiques discernées. Celle-ci est davantage aboutie si les lecteurs adoptent un régime de lecture rationalisé, à la fois conscient et réflexif. Confrontés à une occurrence présumée métonymique, ils recourent alors à divers jugements pour en déceler les motivations et la pertinence stylistique. L'approche modulaire permet d'intégrer les paramètres engagés lors de cette réception métonymique rationnalisée dans un traitement convergent, tout en prenant en compte la variété de ses manifestations. stylistiques à travers lesquelles ils sont à même de mesurer les contributions expressives de ces occurrences aux textes qui les renferment. En tant qu'événements herméneutiques, de telles inférences sont foncièrement dialectiques, mettant en jeu un va-et-vient d'hypothèses, proches de l'abduction de Peirce (1978), en fonction de la spécificité des occurrences rencontrées. Mais ces inférences assurent leur stylisation à travers deux démarches conjointes qui fournissent des sous-modules d'analyse en interaction étroite dans le cadre de leur réception: celles d'identification et d'interprétation.

\section{Identifier la nature des occurrences métonymiques}

Pour que les occurrences métonymiques donnent lieu à des effets de style significatifs à la lecture, leur nature profonde doit être identifiée au préalable. Cette identification consiste à les situer par rapport à certaines matrices rhétoriques implicites régulant la mise en discours des textes. L'objectif est d'éclairer la singularité événementielle des occurrences perçues par des schèmes plus généraux stockés en mémoire. Dans le cas de la métonymie, il s'agit de schèmes cotopiques ${ }^{14}$ selon lesquels il est possible de désigner 
rhétoriquement une entité A par une entité B qui lui est contiguë. Ainsi, derrière l'énoncé de Voltaire cité dans la première partie : "Nous allons chercher à la Chine de la terre ", on reconnaît le type métonymique de la matière pour le produit, souvent attesté dans les énoncés. Ou en-deçà de l'hémistiche de Corneille déjà mentionné : «La main n'est pas le cœur ", on détecte le type métonymique de l'organe pour sa fonction, indéfiniment actualisable dans les productions verbales ${ }^{15}$. Plus concrètement, l'identification d'une occurrence métonymique revient :

40 1) à en repérer le pivot A qui émerge à la surface de l'énoncé ;

2) à déterminer le référent contigu $B$ auquel renvoie ce pivot $A$;

3) à évaluer le schème cotopique sous-jacent $A-B$ sur lequel opère le transfert métonymique particulier de l'occurrence observée.

41 Ces procédures peuvent être testées sur un exemple romanesque, notre analyse ne dépassant pas les contours de notre lecture. Soit ce bref extrait de La Cavale (p. 103) d'Albertine Sarrazin :

Je fredonne en attendant le poste. Jane a piqué à la machine toute la journée, je n'ai guère vu que son dos. Peu à peu, j'ai fumé toutes mes « fringues ».

42 Cet extrait se signale d'emblée par la mise entre guillemets du mot «fringues ». Cette marque de distanciation fonctionne comme un indice typographique intentionnel à notre adresse, de sorte que nous concentrions notre attention sur ce terme mis en relief. Nous sommes encore frappés par l'étrangeté lexicale de la formulation «j'ai fumé toutes mes "fringues" ». Ces indices nous servent de fil conducteur pour tenter d'identifier la nature de la saillance discursive ainsi produite, dont on pourrait se borner à remarquer le relief dans un régime de lecture rapide. Notre compétence linguistique nous confirme la tension sémantique affectant cette formulation suite à l'association d'un verbe + tabagique et d'un nom - tabagique. Par ailleurs, grâce à notre compétence communicationnelle, nous repérons sans peine une liberté prise avec la maxime de modalité de Grice (1979), du fait de l'opacité de cette formulation. D'autres calculs identificatoires nous permettent cependant d'élaborer des hypothèses plus constructives pour élucider son statut. À travers notre compétence textuelle, nous sommes en mesure de nous remémorer qu'elle apparait onze pages après un passage faisant allusion à la pratique du troc par la narratrice en prison :

J'ai des pipes à gogo moi. Mon dernier troc m'a fait un peu de mal au cœur : c'était

le cardigan que je portais la nuit de l'accident, presque mon linceul. (ibid., p. 92)

En sollicitant notre compétence logique, nous restituons dès lors la cohérence de la séquence tabagique relatée :

La narratrice fume des cigarettes achetées avec l'argent obtenu par le troc de ses vêtements.

Si nous rationalisons davantage notre lecture, nous pouvons recourir à notre compétence rhétorique pour voir si «j'ai fumé toutes mes “fringues" " n'est qu'un événement épisodique de discours ou si cette proposition s'enracine sur des schèmes plus profonds. Nous inférons finalement qu'elle s'appuie sur une matrice cotopique Produit (cigarettes) - Instrument (avec l'argent des fringues) pour transférer le second sur le premier (Fringues/Cigarettes). À l'issue d'un raisonnement inductif, un tel transfert ne paraît en rien limité à cette occurrence, mais répondre à des pratiques réitérées, ne serait-ce que parce qu'on le retrouve plus loin dans le même roman dont il constitue l'un des stylèmes : « la peau de bête [...] risque de partir en fumée gauloise par voie de troc » 
(p. 152) ; « cette femme qui souffle mon pécule par les narines » (p. 170); «c'est marrant de fumer un cardigan» (p. 208). Grâce à notre compétence rhétorique technique, nous identifions plus exactement dans ces occurrences un type de métonymie.

Dans l'extrait littéraire de La Cavale, le passage d'un événement stylistique particulier à la reconnaissance d'une matrice rhétorique est facilité par la logique narrative du genre "récit» dans lequel il s'effectue. Mais l'identification d'une métonymie est parfois problématique. Cela arrive lorsque la figure manque d'indices et que les termes contigus qu'elle implique ont une solidarité mouvante entre eux. Ainsi, dans cet exemple poétique de Hugo :

Allons, guerriers ! la charge sonne !

(« La mêlée », Odes et ballades),

est-on en présence d'un transfert du temps sur l'agent ([Pendant] la charge, on sonne $\rightarrow$ Charge/On), de la cause sur l'agent ([À cause de] la charge on sonne $\rightarrow$ Charge/On) ou du but sur l'agent (On sonne [pour] la charge $\rightarrow$ Charge/On) ${ }^{16}$ ? De même, la nature fortement idiolectale de certaines occurrences peu éclairées par leur contexte entrave la perception du type métonymique qu'elles actualisent. La singularité extrême de l'événement stylistique freine à ce moment sa catégorisation, comme dans l'énoncé suivant où il n'est pas aisé d'identifier une métonymie de l'effet (fumée) pour la source (locomotive) :

Une fois par semaine, La Fumée de la Savane traversait la brousse, conduite par des Européens.

(Ousmane, Les Bouts de bois de Dieu, p. 128)

À cela s'ajoute l'ambiguïté d'autres occurrences qui oscillent entre une identification métonymique ou métaphorique, à l'image du début de la description de Paris dans Ferragus (p. 13) de Balzac:

Il est dans Paris certaines rues déshonorées autant que peut l'être un homme coupable d'infamie, puis il existe des rues nobles, puis des rues simplement honnêtes [...]; des rues ouvrières et travailleuses, mercantiles. A-t-on affaire ici à des métonymies du cadre pour ses occupants, la figure se concrétisant sur les rues, ou à des personnifications de ces dernières par des métaphores adjectivales?

\section{Interpréter la sur-signification des occurrences métonymiques}

L'identification des occurrences métonymiques s'accompagne d'une seconde démarche, plus décisive : celle de leur interprétation. Par sa saillance même, une figure comme la métonymie concentre des sens pluriels en attente d'activation. Elle demande par conséquent l'investissement interprétatif des lecteurs afin qu'ils coconstruisent, à leur niveau, ses potentialités informatives en fonction des enjeux et des orientations du texte littéraire produit. À partir de leurs dispositions réceptives et des indices discursifs présentés précédemment, cette activité interprétative consiste en des inférences destinées à expliciter un certain nombre d'informations dont une approche modulaire met à jour la convergence et la diversité dans le cadre de chaque lecture. Ces informations sont énonciatives quand elles font connaître l'attitude du producteur d'une figure (engagement, jugements de valeur...). Elles sont référentielles lorsqu'elles s'appliquent à l'ancrage spatio-temporel ou désignatif de celle-ci. Elles peuvent être encyclopédiques quand elles ont pour objet les univers conceptuels impliqués dans un événement figural. Elles sont encore typologiques, portant sur le genre textuel ou les registres discursifs recouvrant ce dernier. Ces informations sont enfin connotatives, pour 
peu qu'elles offrent des éclairages complémentaires (esthétiques, sociolectaux...) sur tel ou tel aspect d'un énoncé figural. Tout comme pour la phase d'identification des figures, l'activation de ces inférences interprétatives constitue un événement herméneutique en raison de son imprévisibilité et de la variabilité de ses procédures suivant les occurrences rencontrées. En effet, outre qu'il est difficile d'en pressentir le déroulement, elle occasionne des chevauchements de lecture et des retours en arrière toujours possibles. De plus, l'aboutissement de ces inférences s'avère très fluctuant.

51 En ce qui concerne les occurrences métonymiques, le cumul de ces inférences interprétatives permet d'en dégager la sur-signification, à savoir la richesse de leur contenu expressif. Cette cristallisation de significations en leur sein, elle-même inhérente à chaque occurrence, concourt à l'élaboration et à la réception d'un sens global exclusif qui transcende le sens lexicalisé de leurs constituants. Par ailleurs, l'émergence progressive de ce sens global à la lecture parachève la stylisation des occurrences identifiées, en ce qu'elle en dévoile la densité sémantique. De la sorte, une telle approche dynamique du style rejoint les stylistiques attentives à la productivité figurale, illustrées par Paulhan (1977 : 308) : «Le style figuré [...] double en quelque façon la richesse des langues" ".

Si du fait de leur limitation à des transferts par contiguïté, les occurrences métonymiques semblent moins prédisposées à la sur-signification que les occurrences métaphoriques fondées sur la puissance du principe analogique, elles n'en activent pas moins des inférences interprétatives originales, comme le montre cet extrait poétique de Claudel :

C'est la dent que nous mettons à la terre même avec le fer que nous y plantons, et déjà notre pain y mange à la façon dont nous allons le manger. [...] Nous ouvrons d'un soc fort dans la terre solide la raie où naît la croûte que nous coupons de notre couteau et que nous broyons entre nos mâchoires. ( "Le riz », Connaissance de l'Est)

On remarque avant tout deux occurrences saillantes dans la compacité de ce texte : «la dent que nous mettons à la terre » et « dans la terre solide la raie où naît la croûte ». Leur contextualisation insolite révèle des tensions sémantiques qui ne manquent pas de provoquer le questionnement du lecteur. Moyennant des inférences sur le cycle céréalier, il est toutefois à même de déceler leur fonctionnement. Constituant respectivement l'instrument organique de la consommation et l'un des produits cibles de l'univers céréalier, la dent et le pain sont projetés dans la phase initiale de la semence, désignant le grain de blé au terme de connexions métonymiques ${ }^{17}$. De surcroît, les transferts métonymiques par contiguïté se doublent ici de transferts métaleptiques établissant une vision prospective sur le devenir du grain et d'une synecdoque particularisante (croûte/ pain) pour la seconde occurrence :

Semer grain (N1) > Faire farine (N2) avec grain (N1) > Faire pain (N3) avec farine (N2)

$>$ Manger pain (croûte) (N3) avec dent(s) (N4) $\rightarrow$ Dent (N4) et croûte (N3) / Grain (N1)

L'événement de discours inédit constitué par ces transferts métonymiques invite le lecteur à inférer les effets de sens qui en découlent, ce qui conduit à leur stylisation. D'une part, sur le plan référentiel, de telles métonymies se traduisent par une condensation informative de l'énoncé puisque le grain, la dent et la croûte sont prédiqués simultanément. En résulte une forte osmose dénotative dans la description du cycle céréalier, ainsi homogénéisé. D’autre part, sur le plan encyclopédique, ces métonymies suscitent des conflits conceptuels qui modifient notre représentation du monde. Non seulement elles refonctionnalisent la dent et la croûte en éléments originels du cycle céréalier, mais elles les reconceptualisent, en ce qu'elles les assimilent au domaine 
végétal de la semence. De plus, ces métonymies engendrent divers effets stylistiques liés à leur mise en texte. Entre autres, on découvre que la métonymie initiale est aussi motivée par une analogie d'essence métaphorique, la ressemblance de forme entre la dent et le grain favorisant la substitution de la première au second. De même, la disposition de ces métonymies en attaque du poème crée d'emblée une focalisation sur la concision du cycle menant du grain au pain, laquelle contraste avec la profusion du cycle du riz décrit par la suite. Plus largement, sur le plan typologique, il est possible de rendre compte de la vitalité de telles métonymies par le genre poétique dans lequel elles prennent place et à travers lequel le poète Claudel entremêle des notions disparates. En somme, par leur nouveauté et la complexité de leur contenu, ces occurrences métonymiques alimentent une interprétation ouverte et jamais définitive.

Dans certains textes, la sur-signification attachée aux occurrences métonymiques déborde le cadre local d'un passage pour se diffuser sur l'ensemble d'un chapitre. Un exemple représentatif de cette diffusion est fourni par le chapitre VI de la première partie de L'Étranger de Camus. Ce chapitre est ponctué par la métonymie du soleil pour désigner une notion contiguë. Or on détecte sous la répétition de ce terme figuré au moins quatre sens différents suggérés par les cotextes dans lesquels il émerge. Dans une première occurrence, la mention de l'« éclat» du soleil active en celui-ci le sémantisme de la lumière, selon une métonymie Source astrale/Effet visuel :

Le soleil tombait presque d'aplomb sur le sable et son éclat sur la mer était insoutenable. (p. 85)

Six pages plus loin, le cotexte de chaleur mène à l'interprétation d'une métonymie voisine Source astrale/Effet thermique, le soleil étant investi par le sémantisme de la canicule :

Je suis resté devant la première marche, la tête retentissante de soleil. [...] La chaleur était telle qu'il m'était pénible aussi de rester immobile. (p. 91)

Par la suite, avec le meurtre de l'Arabe qui se noue, la métonymie du soleil endosse des sens davantage spécifiques. D’abord, celui de " plage », suscité par la formulation « tout le corps au soleil », au prix d'un transfert Source astrale/Cadre associé :

Le type de Raymond [...] reposait sur le dos, les mains sous la nuque, le front dans

les ombres du rocher, tout le corps au soleil ${ }^{18}$. (p. 92)

Puis celui de « révolver », induit par le syntagme «J'ai secoué [...] le soleil », à travers un transfert Source astrale/Objet associé :

La gâchette a cédé [...] et c'est là, dans le bruit à la fois sec et assourdissant, que tout a commencé. J'ai secoué la sueur et le soleil. [...] Alors, j'ai tiré encore quatre fois. (p. 95)

60 Avec ces variations métonymiques sur un même lexème, selon la figure plus générale de l'antanaclase ${ }^{19}$, on assiste à une double stylisation. D'un côté, on constate un creusement sémantique du terme "soleil », chacune de ses occurrences constituant un événement stylistique en soi. D'un autre côté, ce creusement sémantique s'effectue en corrélation avec les séquences successives du chapitre: si celles-ci réindexent par irradiation le sémantisme du pivot métonymique "soleil», ce dernier symbolise chaque fois leur orientation thématique.

\section{Conclusion}

61 Notre exploration des méandres de la métonymie, vue comme un cas typique de figure dans les œuvres littéraires, nous a permis de la revisiter selon la perspective du style 
comme événement. Ainsi, par son statut d'occurrence plus ou moins imprévisible lors de sa production dans les énoncés, elle apparaît comme un événement disruptif saillant, jouant sur des transferts de désignation entre des termes référentiellement contigus. Néanmoins, sa stylisation progressive intervient essentiellement lors de sa réception par les lecteurs qui mobilisent des procédures d'identification et d'interprétation. C'est en effet à l'issue de différentes inférences de la part de ces derniers que ses effets fonctionnels, cognitifs et esthétiques sont pleinement activés d'après les pactes de lecture inscrits dans les textes. Du fait de son caractère aléatoire au gré de chaque occurrence, ce travail de réception constitue à son niveau un événement herméneutique.

Par ailleurs, cette approche événementielle d'une figure de style comme la métonymie mobilise une dialectique complexe reposant sur la conciliation instable de polarités antagonistes. Locales par leur dimension microstructurale, les métonymies n'acquièrent leur sens irréductible que si l'on considère la globalité de leur contexte. Singulières dans leur actualisation à la surface des textes, elles tendent à une certaine régularité si on les met en relation avec les schèmes rhétoriques qui les sous-tendent. Factuelles dans leur émergence à l'intérieur des textes, elles ne trouvent leur pertinence qu'à travers leur interprétation. Enfin, leurs manifestations diversifiées peuvent être appréhendées d'une manière unitaire si on les analyse dans un cadre modulaire. Par-delà le cas de la métonymie, on a tout à gagner à étendre une telle approche phénoménologique, au plus près de la réalité textuelle, à l'ensemble du style littéraire, trop souvent envisagé d'une façon parcellaire et réductrice.

\title{
BIBLIOGRAPHY
}

\author{
Auteurs cités \\ Aragon, Louis (1946) : Le Crève-cœur, Paris, Gallimard. \\ Balzac, Honoré de (1966 [11833]) : Ferragus, dans La Comédie humaine, t. 4, Paris, Seuil, \\ «L'Intégrale ». \\ Camus, Albert (1942) : L'Étranger, Paris, Gallimard. \\ Claudel, Paul (1929) : Connaissance de l'Est, Paris, Gallimard. \\ Corneille, Pierre (1970 [ $\left.\left.{ }^{1} 1675\right]\right)$ : Suréna, dans Théâtre complet, t. 3, Paris, Classiques Garnier. \\ Diderot, Denis (1962 [171796]) : La Religieuse, dans CEuvres romanesques, Paris, Classiques Garnier. \\ Diderot, Denis (1966 [11805]) : Le Neveu de Rameau, Paris, Gallimard. \\ Diderot, Denis (1972 [1748]) : Les Bijoux indiscrets, Paris, Librairie générale française. \\ Du Bellay, Joachim (1979 [1558]) : Les Regrets, Genève, Droz. \\ Flaubert, Gustave (1998 [11887-1893]) : Correspondance, Paris, Gallimard, « Folio ». \\ Giono, Jean (1931) : Le Grand troupeau, Paris, Gallimard. \\ Hugo, Victor (1972 [ $\left.\left.{ }^{1} 1828\right]\right)$ : Odes et ballades, dans Poésies, t. 1, Paris, Seuil, « L’Intégrale ».
}


Hugo, Victor (1972 [ $\left.\left.{ }^{1} 1880\right]\right)$ : L'Âne, dans Poésies, t. 2, Paris, Seuil, « L'Intégrale ».

Moutot, Michel (2018) : Séquoias, Paris, Seuil.

Ousmane, Sembene (1960) : Les Bouts de bois de Dieu, Paris, Presse-Pocket.

Ousmane, Sembene (1973) : Le Docker noir, Paris, Présence Africaine.

Queneau, Raymond (1936) : Les Derniers jours, Paris, Gallimard.

Queneau, Raymond (1959) : Zazie dans le métro, Paris, Gallimard, « Folio ».

Queneau, Raymond (1969) : Chêne et chien, Paris, Gallimard.

Sarrazin, Albertine (1965) : La Cavale, Paris, Jean-Jacques Pauvert.

Voltaire (1960 [11772]), Les Lettres d'Amabed, dans Romans et contes, Paris, Classiques Garnier.

Voltaire (1967 [11764]) : Dictionnaire philosophique, Paris, Classiques Garnier.

Voltaire (1973 [ $\left.\left.{ }^{1} 1765\right]\right)$ : Questions sur les miracles, dans Facéties, Paris, PUF, « À la découverte ».

Zola, Émile (1979 [11892]) : La Débâcle, Paris, Fasquelle.

\section{Références théoriques}

Adam, Jean-Michel (1997) : Le Style dans la langue, Lausanne/Paris, Delachaux \& Niestlé.

Apothéloz, Denis (1995) : Rôle et fonctionnement de l'anaphore dans la dynamique textuelle, Genève, Droz.

Benveniste, Émile (1966) : Problèmes de linguistique générale, Paris, Gallimard.

Bonhomme, Marc (1999) : "Rhétorique modulaire et figures du discours », in Henning Nølke \& Jean-Michel Adam dirs, Approches modulaires : de la langue au discours, Lausanne/Paris, Delachaux et Niestlé, p. 163-186.

Bonhomme, Marc (2006) : Le Discours métonymique, Berne, Peter Lang.

Bonhomme, Marc (2014) : Pragmatique des figures du discours, Paris, Honoré Champion.

Borel, Marie-Jeanne, Grize, Jean-Blaise \& Miéville, Denis (1983) : Essai de logique naturelle, Berne, Peter Lang.

Cohen, Jean (1970) : « Théorie de la figure », Communications, n 16, p. 3-25.

Détrie, Catherine (2001) : Du sens dans le processus métaphorique, Paris, Honoré Champion.

Ducrot, Oswald (1979) : « Les lois de discours », Langue française, $n^{\circ}$ 42, p. 21-33.

Dumarsais, César Chesneau (1967 [11756]) : « Figure », Encyclopédie, t. 6, Stuttgart, Friedrich

Frommann, p. 766-772.

Dumarsais, César Chesneau (1977 [11730]) : Traité des tropes, Paris, Le Nouveau Commerce.

Dupriez, Bernard (1984) : Gradus, Paris, Union Générale d'Éditions, « 10-18 ».

Fontanier, Pierre (1968 [ ${ }^{1} 1821$ et 1827]) : Les Figures du discours, Paris, Flammarion.

Foucault, Michel (1969) : L'Archéologie du savoir, Paris, Gallimard.

Fouquelin, Antoine (1990 [11555]) : La Rhétorique françoise, in Traités de poétique et de rhétorique de la Renaissance (éd. F. Goyet), Paris, Librairie Générale Française, p. 345-464.

Gardes Tamine, Joëlle (2011) : Pour une nouvelle théorie des figures, Paris, PUF.

Grice, Henri Paul (1979) : « Logique et conversation », Communications, n 30, p. 57-72. 
Groupe $\mu$ (1970) : Rhétorique générale, Paris, Larousse.

Herschberg-Pierrot, Anne (2005) : Le Style en mouvement, Paris, Belin.

Jakobson, Roman (1963) : Essais de linguistique générale, Paris, Minuit.

Kerbrat-Orecchioni, Catherine (1994) : « Rhétorique et pragmatique : les figures revisitées »,

Langue française, $n^{\circ} 101$, p. 57-71.

Lamy, Bernard (1675) : La Rhétorique ou l'art de parler, Paris, chez André Pralard.

Le Guern, Michel (1973) : Sémantique de la métaphore et de la métonymie, Paris, Larousse.

Longin (1993) : Du sublime, Paris, Payot et Rivages.

Meyer, Michel (2008) : Principia rhetorica, Paris, Fayard.

Paulhan, Jean (1977) : Traité des figures, Paris, Le Nouveau Commerce.

Peirce, Charles Sanders (1978) : Écrits sur le signe, Paris, Seuil.

Perelman, Chaïm \& Olbrechts-Tyteca, Lucie (1988) : Traité de l'argumentation, Bruxelles, Éditions de l'Université de Bruxelles.

Plantin, Christian (2009) : " Un lieu pour les figures dans la théorie de l'argumentation », Argumentation et analyse du discours, $\mathrm{n}^{\circ} 2$, http://journals.openedition.org/aad/215 [consulté le $10 / 10 / 2018]$.

Quine, Willard van Orman (1951) : Mathematical Logic, Cambridge, Harvard University Press.

Quintilien (1978) : Institution oratoire, t. 5, Paris, Les Belles Lettres.

Rabatel, Alain (2008) : «Figures et points de vue en confrontation ", Langue française, $\mathrm{n}^{\circ} 160$, p. 3-19.

Recanati, François (1979) : La Transparence et l'énonciation, Paris, Seuil.

Riffaterre, Michael (1971) : Essais de stylistique structurale, Paris, Flammarion.

Sabot, Philippe (2017) : « Le statut de l'événement dans la pensée de Michel Foucault : des Mots et les choses à L'Archéologie du savoir », communication faite dans le cadre du séminaire « Actualités de Foucault » en décembre 2016, https//hal.univ-lille3.fr/hal-01517790 [consulté le 17-10-2018].

Tamba-Mecz, Irène (1981) : Le Sens figuré, Paris, PUF.

Todorov, Tzvetan (1967) : Littérature et signification, Paris, Larousse.

\section{NOTES}

1. On trouvera dans Bonhomme (1999 et 2014) une vision d'ensemble sur cette conception modulaire des figures de style.

2. Pour plus de détails sur cette figure majeure du discours, voir Le Guern (1973) ou Bonhomme (2006).

3. "L'auteur réaliste opère des digressions métonymiques de l'intrigue à l'atmosphère et des personnages au cadre spatio-temporel. Il est friand de détails synecdochiques » (Jakobson 1963 : 63).

4. En cela, nous nous situons dans la mouvance de la pragmatique énonciative de Benveniste (1966) et de Recanati (1979 : 153) pour lequel « le fait de dire quelque chose est un événement ». 
5. Dans une optique similaire, voir aussi Dupriez (1984:9) au sujet des figures en général : « Les figures sont la forme [...] de ce surgissement, souvent indifférencié, du moi au monde ».

6. Cette terminologie est de Dumarsais (1977). Les métonymies du signe se distinguent par leur dimension concrète et emblématique, comme dans les deux exemples relevés où des réalités abstraites (le mariage, l'amour et la parole) sont respectivement désignées par les parties du corps (la main, le cœur et la langue) qui leur sont associées référentiellement.

7. Comme l'écrit le Petit Robert (2016: 960), l'événement est «ce qui a quelque importance pour l'être humain ".

8. Par ses décalages de qualités entre deux notions contiguës, l'hypallage forme une «figure paramétonymique » (Bonhomme $2006: 61$ ).

9. On peut encore voir une syllepse de sens dans l'adjectif «blanc», en ce que son sémantisme chromatique est ici associé au sème afférent/silence/, à travers un renvoi implicite à la formule " un blanc dans la conversation ».

10. Ces deux figures se rangent en effet dans la «polarité métonymique » (Jakobson $1963: 61$ ) du langage. La synecdoque constitue une métonymie variationnelle par ses transferts particularisants ou généralisants. La métalepse est définissable comme une métonymie chronologique par ses transferts prospectifs ou rétrospectifs.

11. Ces formulations métonymiques sont en effet utilisées à la fois en usage et en mention. Dans notre analyse, nous reprenons la distinction classique de Quine (1951) entre l'usage d'un signe en contexte standard et sa mention en contexte métalinguistique.

12. Pour ce concept, voir Apothéloz (1995).

13. On aborde ici la facette constructiviste de l'événement, formulée en ces termes par Sabot (2017 : 5) : «Rupture et ouverture sont les deux caractères majeurs d'un "événement" qui est moins ce qui a lieu (dans des coordonnées spatio-temporelles déterminées) que ce qui donne lieu, ce qui ouvre un nouvel espace de pensée ».

14. Liés à la logique naturelle (Borel, Grize \& Miéville 1983), les schèmes cotopiques sont des réseaux conceptuels associant des notions compatibles dans un univers référentiel donné.

15. On retrouve, au niveau d'une figure comme la métonymie, l'idée exprimée par Foucault (1969) selon laquelle, loin d'être purement évanescent, l'événement s'ouvre à une existence rémanente en s'offrant à la répétition.

16. Par sa polyvalence fonctionnelle, source d'indécidabilité, une telle configuration métonymique constitue manifestement un "plateau multistable», au sens que Mathilde Vallespir donne à cette formulation dans sa contribution au présent numéro.

17. En plus, la dent peut désigner l'instrument de la semence, à savoir le soc.

18. Cette occurrence est cependant relativement lexicalisée, ce qui la rend moins saillante que les autres.

19. Constituant une forme de polysémie discursive, l'antanaclase qualifie les bifurcations sémantiques d'un mot répété dans un énoncé.

\section{ABSTRACTS}

La présente étude questionne l'articulation entre le style littéraire et le concept d' « événement » à travers le cas d'une des figures du discours les plus représentatives : la métonymie. Celle-ci est redéfinie comme un "événement de style » en ce qu'elle constitue une intrusion à la fois 
déstabilisante et hautement signifiante à la surface des textes. Dans un premier temps, la production de métonymies est décrite comme un événement de style disruptif en raison des ruptures communicationnelles, des saillances textuelles et des tensions sémantiques que leur émergence suscite au sein des occurrences littéraires. Dans un second temps, on s'intéresse à la réception des énoncés métonymiques, envisagée comme un événement de style herméneutique $\mathrm{du}$ fait $\mathrm{du}$ caractère aléatoire de leur identification et de leur interprétation selon les particularismes des différents contextes. Par ailleurs, on analyse l'activité de stylisation des lecteurs qui consiste à donner du sens et une pertinence esthétique à la singularité des métonymies identifiées. Au total, une telle approche événementielle d'une figure comme la métonymie met en évidence son irréductibilité discursive en fonction des pactes de lecture instaurés par chaque texte littéraire.

\section{INDEX}

Mots-clés: émergence, événement de style, métonymie, sur-signification, stylisation

\section{AUTHOR}

\section{MARC BONHOMME}

Université de Berne

Docteur d'État-ès-Lettres et agrégé de grammaire, Marc Bonhomme est professeur émérite de linguistique française à l'Université de Berne. Il est notamment l'auteur des Figures clefs du discours (Paris, Seuil, coll. « Mémo », 1998), du Discours métonymique (Berne, Peter Lang, 2006), de L'Argumentation publicitaire - avec Jean-Michel Adam - (Paris, Armand Colin, 2012) et de Pragmatique des figures du discours (Paris, Honoré Champion, 2014). Il a récemment codirigé Métaphore et argumentation - avec Anne-Marie Paillet et Philippe Wahl - (Louvain-la-Neuve, Academia, « Au cœur des textes », 2017), ainsi que La Présupposition entre théorisation et mise en discours - avec Amir Biglari - (Paris, Classiques Garnier, « Série linguistique », 2018). Il a aussi publié de nombreux articles dans les domaines de la stylistique, de la rhétorique, de l'histoire de la langue française et de l'analyse du discours. Il prépare actuellement une édition critique des Observations sur la langue françoise (1675-1676) de Gilles Ménage pour les Classiques Garnier.- 